\title{
Electrically-tunable optical zoom system by using liquid crystal lenses
}

\author{
Yi-Hsin Lin*, Ming-Syuan Chen and Hung-Chun Lin \\ Department of Photonics, National Chiao Tung Unversity, 1001 Ta Hsueh Rd., Hsinchu 30010, \\ Taiwan \\ *Corresponding author: yilin@mail.nctu.edu.tw
}

\begin{abstract}
An electrically-tunable optical zoom system using liquid crystal (LC) lenses is demonstrated. The mechanism of the optical zoom system is to use two lenses and a camera system to achieve focusing and zooming function. In this paper, we analyzed the imaging conditions and the magnification of the optical zoom system. The relation between the focusing properties of LC lenses and zoom ratio of the optical zoom system is also discussed. The electrically-tunable optical zoom system using two LC lenses has high zoom ratio $(\sim 7.9: 1$ to $\sim 5.5: 1)$, short system length $(<10 \mathrm{~cm})$ and the object can be zoomed in or zoomed out continuously at the objective distance of infinity to $10 \mathrm{~cm}$. The potential applications are cell phones, cameras, telescopes and pico projectors.
\end{abstract}

Keywords: Liquid-crystal devices; Liquid-crystal lenses; Non-mechanical optical zoom.

\section{INTRODUCTION}

Electrically tunable optical zoom systems are essential for portable optical applications, such as cell phones, cameras, pico projectors and the night vision of hand-carried weapons ${ }^{1-3}$. Traditional optical zoom systems which consist of many solid lenses, delicate driving motors and an image sensor are bulky, complicated, and fragile. To realize electrically tunable optical zoom system, several active-focus elements can be adopted, such as liquid lenses ${ }^{1-2}$, deformable mirrors ${ }^{3}$, and liquid crystal (LC) lenses ${ }^{4-7}$.The features of LC lenses are low cost, light weight, and no mechanical moving part. Tam proposed and did a theoretical analysis of electro-optical zoom lenses based on two spatial light modulators and two solid lenses, but did not show the experimental results ${ }^{7}$. Following the analysis, Ye and Valley also demonstrated the electro-optical zoom system. ${ }^{8-9}$ However, the disadvantages of the proposed designs are the discrete magnifications, fixed location of an object, large size of the system $(>50 \mathrm{~cm})$, and a small zoom ratio $(\sim 2: 1)$. It is important to realize an electrically tunable-focusing optical zoom system based on LC lenses with a small size of the system, a continuous tunable objective distance and a continuous tunable image magnification.

Advances in Display Technologies II, edited by Liang-Chy Chien, Sin-Doo Lee, Ming Hsien Wu, Proc. of SPIE Vol. 8280, 82800Q - (C) 2012 SPIE - CCC code: 0277-786X/12/\$18 · doi: 10.1117/12.912063

Proc. of SPIE Vol. 828082800 Q-1 
In this paper, we demonstrate a compact electrically tunable-focusing optical zoom system using two LC lenses. The optical principle in the system is investigated first. To obtain a large zoom ratio, we design a composite LC lens which consists of a sub-LC lens and a planar polymeric lens with a fixed negative lens power. The zoom ratio of the optical zoom system reaches up to $\sim 7.9: 1$ with $10 \mathrm{~cm}$ system length. The object can be zoomed in or zoomed out continuously at the objective distance from infinity to $10 \mathrm{~cm}$. In addition, optical zoom system with $2 \mathrm{~cm}$ system length is also demonstrated. However, the zoom ratio is smaller $(\sim 1.9: 1)$ when the system length is reduced. The potential applications are cell phones, cameras, telescopes and pico projectors. ${ }^{10-11}$

\section{OPERATING MECHANISM}

Figure 1 depicts the structure of the optical zoom system consisting of a target (or an object), two LC lenses, and a camera system which is focused on an infinite distance. The focal length and the lens power, defined as the inverse of focal length, of the LC object lens are $\mathrm{f}_{\mathrm{o}}$ and $\phi_{o}$, respectively. The focal length and the lens power of the LC eyepiece lens are $\mathrm{f}_{\mathrm{e}}$ and $\phi_{e}$, respectively. The distance between the target and the LC object lens is $\mathrm{p}$, the distance between the LC object lens and the LC eyepiece lens is $\mathrm{d}$, and the distance between LC eyepiece lens and the lens is q. A collimated light is incident on the lens in order to collect the incident light into the image sensor.

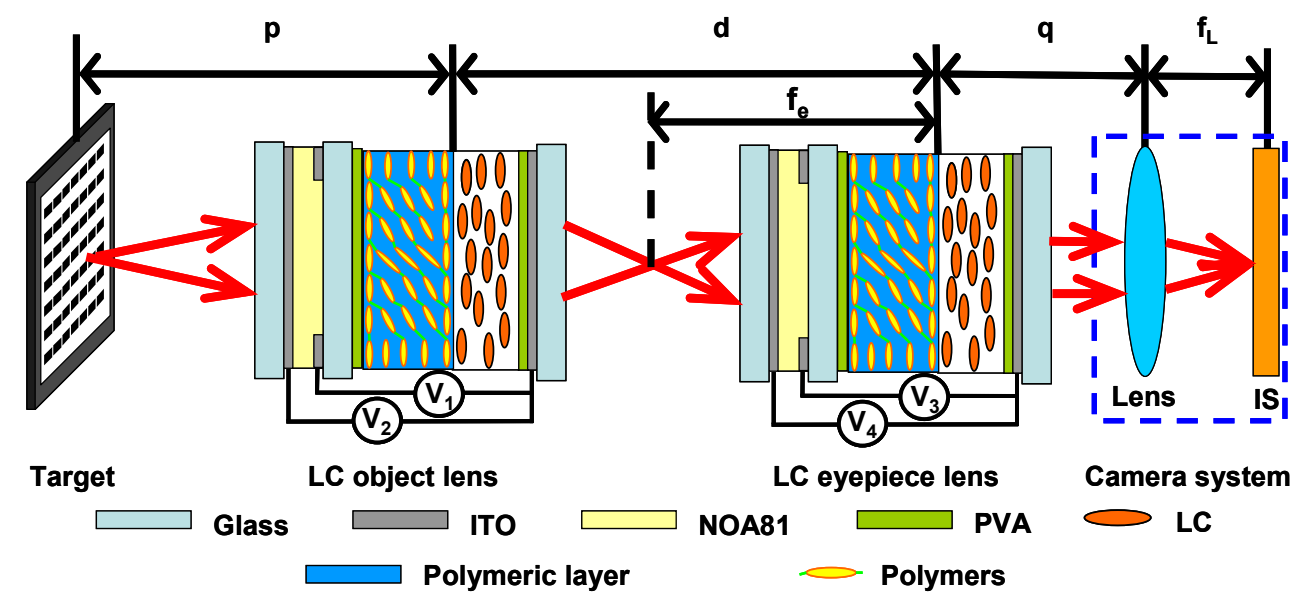

Figure 1 : The structure of the zoom system. IS: image sensor. $\mathrm{f}_{\mathrm{L}}$ : the focal length of the lens.

In order to obtain a collimated light right after the LC eyepiece lens, the relation among $\phi_{o}, \phi_{e}, \mathrm{p}$, and $\mathrm{d}$ should be ${ }^{12}$ : 


$$
\frac{1}{\phi_{e}}+\frac{p}{\phi_{o} \times p-1}=d .
$$

From Eq. (1), the magnification (M) of the optical zoom system in Figure 1 can be written as:

$$
M=\frac{\phi_{e} \times p}{\left(1-\phi_{o} \times p\right)} .
$$

We assume that magnification is positive (i.e the erect image) and the LC lens could be switched as a positive or a negative lens. The lens power of the LC lens is $\phi$ and $\phi$ has a constraint: a minimum $\phi_{\min }$ and a maximum $\phi_{\max }$ (i.e. $\phi_{\min } \leq \phi \leq \phi_{\max }$ ). Both of $\phi_{\min }$ and $\phi_{\max }$ limit the tunable lens power range of the LC lens. The minimum lens power $\phi_{\min }$ is negative, and the maximum lens power $\phi_{\max }$ is positive. In the experiment, the maximum lens power is usually larger than absolute value of minimum lens power (i.e $\left.\phi_{\max }>\left|\phi_{\min }\right|\right)$ under two mode switching of a LC lens. From Eq. (1) and Eq. (2), when $\phi_{o}$ equals $\phi_{\min }$, the system has a minimum magnification $\left(\mathrm{M}_{\min }\right)$ :

$$
M_{\min }=\frac{p}{p+d-d \times p \times \phi_{\min }} .
$$

When $\phi_{e}$ equals $\phi_{\min }$, the system has a maximum magnification $\left(\mathrm{M}_{\max }\right)$ :

$$
M_{\max }=1-d \times \phi_{\min } .
$$

The range of the magnification of the optical zooming system is then limited by $\mathrm{M}_{\max }$ and $\mathrm{M}_{\min }$. The zoom ratio (ZR) of an optical zooming system can be defined as the ratio of $\mathrm{M}_{\max }$ to $\mathrm{M}_{\min }$. From Eq. (3) and Eq. (4), the ZR turns out:

$$
Z R=\left(\phi_{\min } \times d-1-\frac{d}{p}\right) \times\left(\phi_{\min } \times d-1\right) .
$$

From Eq. (5), the zoom ratio of the system is related to three parameters: $\phi_{\min }$, $d$, and $p$. In order to obtain a larger zoom ratio, the LC lenses we used in Figure 1 are the composite LC lenses which have small minimum lens power. The composite LC lens consists of a sub-LC lens and a built-in planar polymeric lens. The lens power of the polymeric lens is negative and fixed. The composite LC lens was operated by two voltages, (i.e. $V_{1}$ and $V_{2}$ or $V_{3}$ and $V_{4}$ in Fig.1). Because the structure of the two LC lenses in the system is identical, we just use the LC object lens to illustrate the LC lens 
properties. The lens power of the composite LC lens $\left(\phi_{c}\left(V_{1}, V_{2}\right)\right)$ can be expressed as:

$$
\phi_{c}\left(V_{1}, V_{2}\right)=\phi_{L C}\left(V_{1}, V_{2}\right)+\phi_{p}
$$

where $\phi_{L C}\left(V_{1}, V_{2}\right)$ is the voltage-dependent lens power of sub-LC lens contributed from the LC layer and $\phi_{p}$ is the

fixed lens power of the polymeric lens. The $\phi_{L C}\left(V_{1}, V_{2}\right)$ depending on the wavelength of light $(\lambda)$, aperture size (w), and phase difference $(\Delta \delta)$ can be written as Eq. (7): ${ }^{12}$

$$
\phi_{L C}\left(V_{1}, V_{2}\right)=\frac{4 \times \lambda \times \Delta \delta\left(V_{1}, V_{2}\right)}{\pi \times w^{2}} .
$$

Therefore, we can control the applied voltages of the composite LC lenses to adjust lens power of the composite LC lenses continuously and then realize an electrically tunable-focusing optical zoom system with large zoom ratio.

\section{EXPERIMENTAL RESULTS AND DISCUSSION}

\subsection{Composite LC lens}

\subsubsection{Focusing ability}

The detail structure of the composite LC lens for LC object lens and LC eyepiece lens in Figure 1 consists of three Indium-Tin Oxide (ITO) glass substrates with thickness of $0.7 \mathrm{~mm}$, an isolating layer (NOA 81, Norland Optical Adhesive) with thickness of $35 \mu \mathrm{m}$, mechanically buffered alignment layers (Polyvinylalcohol or PVA), a polymeric layer with thickness of $35 \mu \mathrm{m}$ and a LC layer with thickness of $50 \mu \mathrm{m}$. The ITO layer in the middle of glass substrate was etched with a hole-pattern within a diameter of $1.28 \mathrm{~mm}$.

To observe the phase profile of two composite LC lenses, we observed the image of the composite LC lenses at different voltages under crossed polarizers. Figure 2(a) shows the images of the composite LC lens. The rubbing direction of the composite LC lens was 45 degree with respect to one of the polarizers. In Figure 2(a), the left-top one is the phase profile of the positive lens with maximum lens power, the right-top one is the phase profile with no lens power, the left-bottom one is the phase profile of the polymeric lens, and the right-bottom one is the phase profile of the negative lens with minimum lens power. The number of concentric rings of Figure 2(a) is proportional to the phase profile of the composite LC lens. We convert the phase profile to the lens power according to the relation: 
$\phi=8 \times \lambda \times N / D^{2}$, where $\mathrm{D}$ is the aperture size, $\lambda$ is the wavelength, $\mathrm{N}$ is the number of rings of the phase profile. The lens powers of two composite LC lenses as a function of applied voltage are shown in Figure 2(b). In Figure 2(b), because of the polymeric layer with lens power $\sim-5.3 \mathrm{~m}^{-1}$, the composite LC lens is a negative lens with lens power $-5.3 \mathrm{~m}^{-1}$ when $\mathrm{V}_{1}=\mathrm{V}_{2}$. When $\mathrm{V}_{2}<80 \mathrm{~V}_{\mathrm{rms}}$ at $\mathrm{V}_{1}=80 \mathrm{~V}_{\mathrm{rms}}$, the lens power of the composite $\mathrm{LC}$ lens is switchable from $-5.3 \mathrm{~m}^{-1}$ to $21.8 \mathrm{~m}^{-1}$. At $\mathrm{V}_{1}=80 \mathrm{~V}_{\mathrm{rms}}$ and $\mathrm{V}_{2}=38 \mathrm{~V}_{\mathrm{rms}}$, the lens power of the LC layer equals the lens power of the polymeric layer and there is no phase difference in the phase profile, as shown in Figure 2(a). As a result, the lens power of the composite LC lens is zero. At $\mathrm{V}_{1}<40 \mathrm{~V}_{\text {rms }}$ and $\mathrm{V}_{2}=40 \mathrm{~V}_{\text {rms }}$, the composite LC lens is a negative lens with switchable lens power from $-13.5 \mathrm{~m}^{-1}$ to $-5.3 \mathrm{~m}^{-1}$. From Figure 2(b), the tunable lens power range of the composite LC lens is from minimum lens power $-13.5 \mathrm{~m}^{-1}$ to maximum lens power $21.8 \mathrm{~m}^{-1}$. It also means the tunable focal length range of the composite LC lens is smaller than $-7.4 \mathrm{~cm}$ or larger than $4.6 \mathrm{~cm}$.
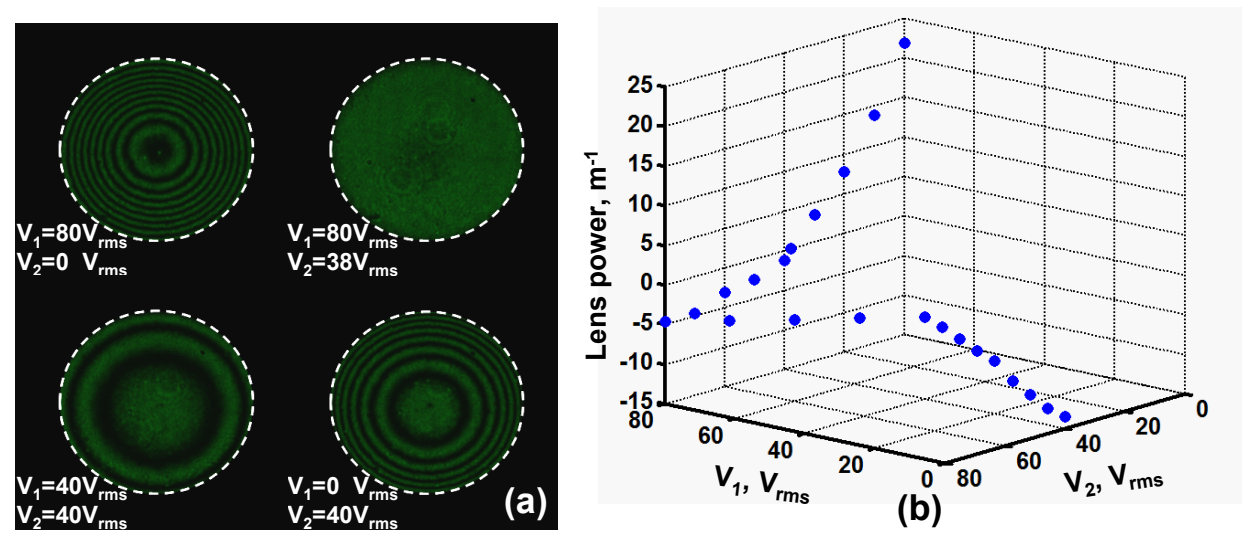

Figure 2. (a) The phase profiles of the composite LC lens at different voltages. (b) The lens power $f_{o}$ the composite LC lens as a function of applied voltages $\mathrm{V}_{1}$ and $\mathrm{V}_{2} \cdot \lambda=532 \mathrm{~nm}$.

\subsubsection{Response time}

The measured response time of the composite LC lens, including the focusing time and defocusing time, is around $3.8 \mathrm{sec}$ when we switched the voltages between $\left(\mathrm{V}_{1}, \mathrm{~V}_{2}\right)=\left(80 \mathrm{~V}_{\mathrm{rms}}, 80 \mathrm{~V}_{\mathrm{rms}}\right)$ and $\left(\mathrm{V}_{1}, \mathrm{~V}_{2}\right)=\left(80 \mathrm{~V}_{\mathrm{rms}}, 0 \mathrm{~V}_{\mathrm{rms}}\right)$, as shown in Figure 3(a) and (b). We also measured the response time of the LC lens without the polymeric lens, as shown in Figure $3(c)$ and (d). The measured response time of the LC lens without the polymeric lens is around $3.8 \mathrm{sec}$. This means the polymeric layer of the composite LC lens does not affect the response time.

Because the thickness of the LC layer of the composite LC lens is fixed, we can assume the response time of the LC lens is similar to the LC homogeneous cell. The rise time $t_{\text {rise }}$ and fall time $t_{\text {fall }}$ of a LC homogeneous cell are $t_{\text {rise }}=t_{\text {fall }} /\left[\left(V_{L C} / V_{t h}\right)^{2}-1\right]$ and $t_{\text {fall }}=\gamma_{1} \cdot d_{L C} /\left(K_{11} \cdot \pi^{2}\right) \cdot{ }^{13} \mathrm{~V}_{\mathrm{LC}}$ is the applied voltage of the LC layer, $\mathrm{V}_{\text {th }}$ is the threshold voltage, $\gamma_{1}$ is the viscosity constant of the LC, $\mathrm{K}_{11}$ is the splay elastic constant of the LC, and $\mathrm{d}_{\mathrm{LC}}$ is the thickness of the $\mathrm{LC}$ layer. $\mathrm{V}_{\mathrm{LC}}$ can be obtained by calculating parallel-plate capacitor of the LC lens. The applied voltage 
of the LC layer of the composite LC lens $\mathrm{V}_{\mathrm{LC}-\text { com }}$ can be written as: ${ }^{14}$

$$
V_{L C-c o m}=V \cdot\left(\frac{d_{L C} \cdot \varepsilon_{g} \cdot \varepsilon_{p}}{d_{L C} \cdot \varepsilon_{g} \cdot \varepsilon_{p}+d_{p} \cdot \varepsilon_{L C} \cdot \varepsilon_{p}+d_{g} \cdot \varepsilon_{L C} \cdot \varepsilon_{p}}\right) .
$$

In Eq. (8), $d_{L C}, d_{p}$ and $d_{g}$ are the thickness of the LC layer, the thickness of the polymeric lens and the thickness of the glass, respectively. $\varepsilon_{\mathrm{LC}}, \varepsilon_{\mathrm{p}}$, and $\varepsilon_{\mathrm{g}}$ are the dielectric constants of the LC layer, the polymeric lens and the glass, respectively. $\mathrm{V}$ is the applied voltage on the electrode. The applied voltage of the LC layer for the LC lens without the polymeric layer $\left(\mathrm{V}_{\mathrm{LC}-\mathrm{nor}}\right)$ can be expressed as:

$$
V_{L C-n o r}=V \cdot\left(\frac{d_{L C} \cdot \varepsilon_{g}}{d_{L C} \cdot \varepsilon_{g}+d_{g} \cdot \varepsilon_{L C}}\right) .
$$

In the experiments, $d_{g} \gg>d_{p}$ and $d_{g} \gg>d_{L C}$. Eq. (8) and Eq. (9) can be simplified as $V \cdot\left[\left(d_{L C} \cdot \varepsilon_{g}\right) /\left(d_{g} \cdot \varepsilon_{L C}\right)\right]$. Therefore, the response time of the composite LC lens almost equals the LC lens without the polymeric layer.
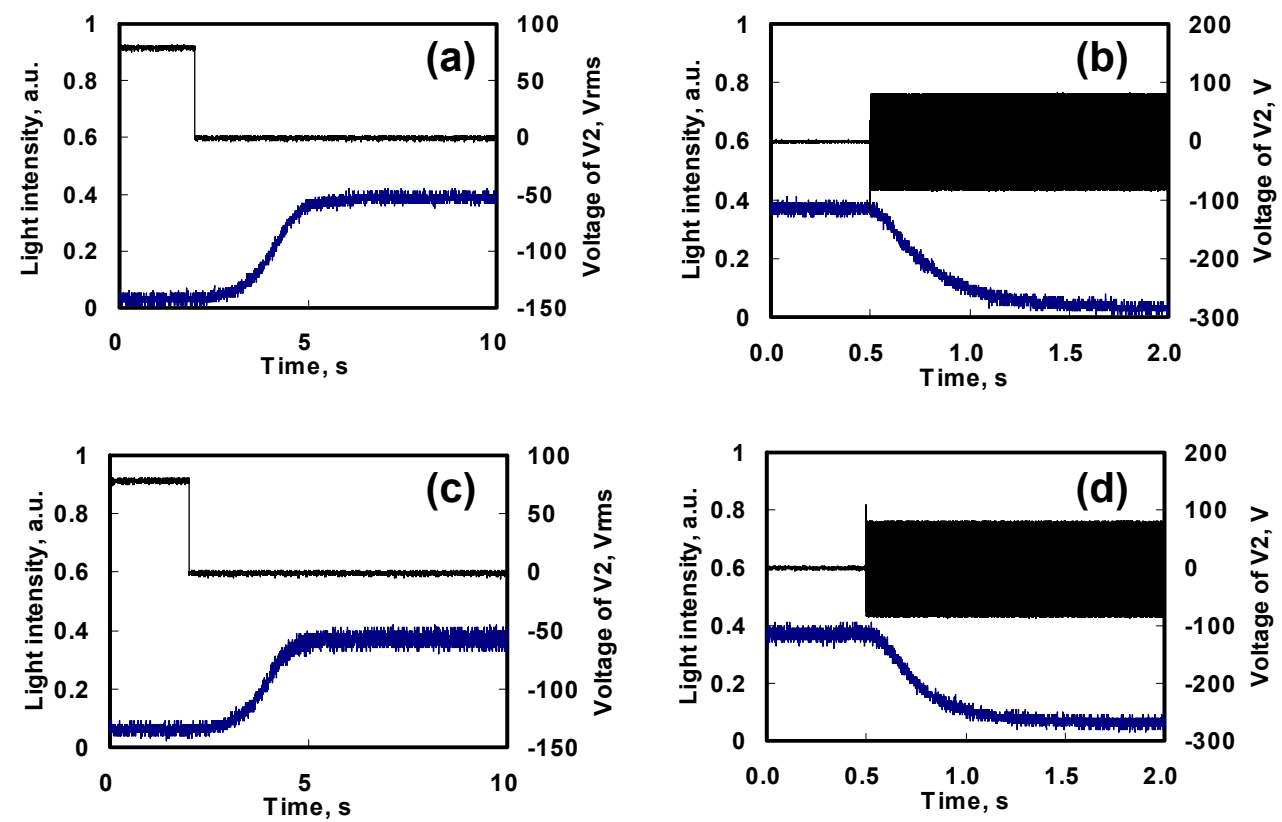

Figure 3. (a) The focusing and (b) de-focusing response of the composite LC lens and the (c) focusing and (d) de-focusing response of the LC lens without the polymeric layer. The black line indicates the applied voltage $\mathrm{V}_{2}$. The blue line indicates the light intensity. $\mathrm{V}_{1}$ $=80 \mathrm{~V}_{\mathrm{rms}}$. 


\subsection{Optical zoom}

To measure the zoom ratio of the system in Figure.1, we attached a polarizer on the LC object lens whose transmissive axis is parallel to the rubbing direction. We also placed a target with black squares with the area of $0.55 \mathrm{~mm}$ $\mathrm{x} 0.55 \mathrm{~mm}$ and then adjusted voltages of two composite LC lenses to obtain the images with different magnifications. By measuring the size change of the central square of the image, we can measure the magnification. The captured images for different $\mathrm{p}$ and $\mathrm{d}$ at $\mathrm{M}=1, \mathrm{M}_{\min }$ and $\mathrm{M}_{\max }$ are shown in Figure 4.

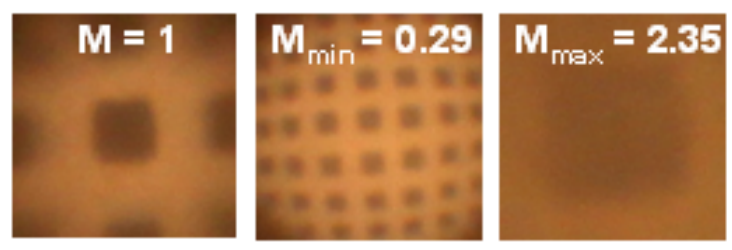

(a)
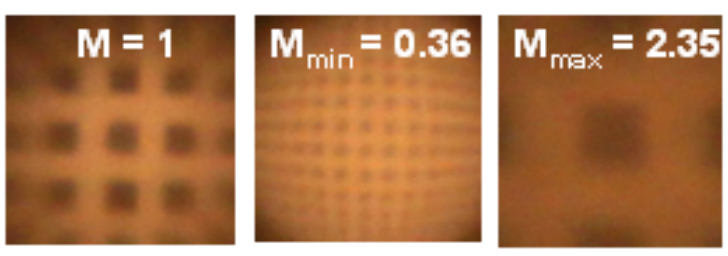

(b)
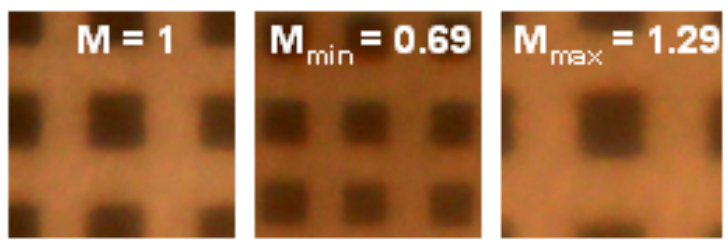

(c)

Figure 4. Images taken with the optical zoom system when (a) $d=10 \mathrm{~cm}, \mathrm{p}=10 \mathrm{~cm}$, (b) $d=10 \mathrm{~cm}, p=20 \mathrm{~cm}$, and (c) $d=2 \mathrm{~cm}, \mathrm{p}=$ $10 \mathrm{~cm}$.

The relation between magnification and the object distance $\mathrm{p}$ is already discussed in the previous published literature.

${ }^{12}$ The magnification as a function of $d$ which is the distance between two LC lenses is shown in Figure 5. The maximum magnification (black dot in Figure 6) decreases from 2.35 to 1.29 and the minimum magnification (blue triangles in Figure 5) increases from 0.29 to 0.69 as d decreases. After putting the experimental parameters to Eq. (4): $\phi_{\min }=-13.5$ $\mathrm{m}^{-1}$ and $\mathrm{p}=10 \mathrm{~cm}$. The theoretical maximum magnification decreases form 2.35 to 1.27 which is closed to the experimental result. From Eq. (3), the minimum magnification increases 0.30 to 0.68 which is also closed to the experimental results. From Figure 5, we can obtain the zoom ratio as function of $d$ as shown in Figure 6(blue dots). The maximum magnification and the minimum magnification approach to 1 when $\mathrm{d}$ decreases. Therefore, the zoom ratio decreases from 7.93:1 to $1.86: 1$ when d decreases from $10 \mathrm{~cm}$ to $2 \mathrm{~cm}$. According to Eq. (5), the theoretical as a function 
of $d$ is also plotted in Figure 6(gray line). The experimental and theoretical results agree well.

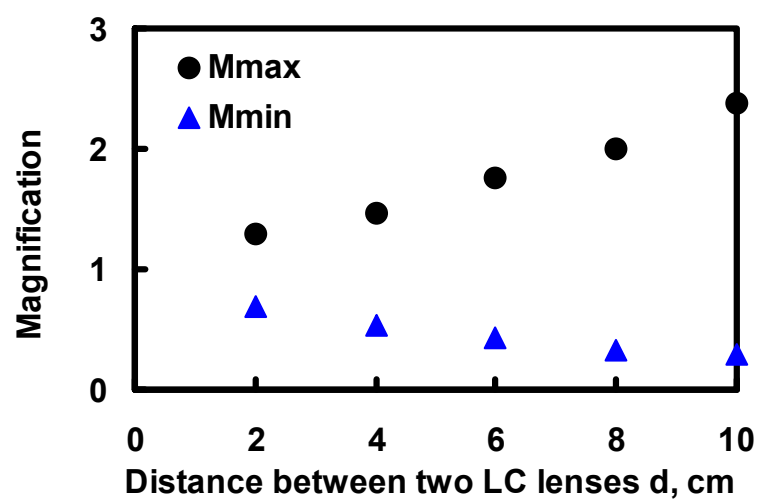

Figure 5. The measured magnification as a function of the distance between two LC lenses (or d). The black dots indicate the maximum magnification and the blue triangles indicate the minimum magnification.

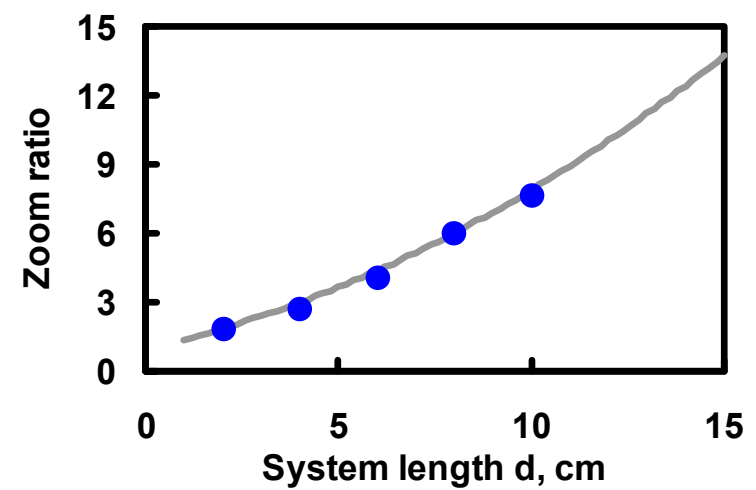

Figure 6. The zoom ratio as a function of the distance between two LC lenses (or d). The blue dots indicate the experimental results and the gray line indicates the simulation results.

\section{CONCLUSION}

We have demonstrated electrically tunable focusing optical zoom system by using two composite liquid crystal lenses. The zoom ratio depending on the location of object is up to $7.9: 1$ when the size of system is $10 \mathrm{~cm}$. The optical zoom system also can be achieved with smaller size compare with the previous designs. When the size of system is $2 \mathrm{~cm}$, the zoom ratio of the system is $\sim 1.9: 1$. The object from infinity to $10 \mathrm{~cm}$ can be zoomed continuously by changing the voltage of two composite LC lenses. Compare with the conventional optical zoom system which needs spaces for optical elements moving, our designed system has a smaller size of the electrically tunable-focusing optical zoom system. However, the zoom ratio of the system decreases when the size of the system decreases. We can increase the tunable lens power range of the LC lenses by improving the birefringence of liquid crystal materials and enlarging the cell gap to 
maintain the zoom ratio large. Enlarging the cell gap would result in the slow response time and the scattering. To improve the light efficiency and image quality, polarizer-free LC lenses with large aperture size and achromatic designs should be developed. The potential applications are cell phones, cameras, telescopes and pico projectors.

The authors acknowledge support from the National Science Council (NSC) in Taiwan under the contract no. 98-2112-M-009-017-MY3.

\section{REFERENCE}

[1] Peng, R., Chen, J., and Zhuang, S., "Electrowetting-actuated zoom lens with spherical-interface liquid lenses," J. Opt. Soc. Am. A 25, 2644 (2009).

[2] Zhang, D. Y., Justus, N., and Lo, Y. H., "Fluidic adaptive zoom lens with high zoom ratio and widely tunable field of view," Opt. Commun. 249, 175 (2005).

[3] Seidl, K., Nnobbe, J., and Grüger, H., "Design of an all-reflective unobscured optical-power zoom objective" Appl. Opt. 48, 4097 (2009).

[4] Wick, D. V., Martinez, T., Payne, D. M., Sweatt,W. C., and Restaino, S. R., “Active optical zoom system,” Proc. SPIE 5798, 151 (2005).

[5] Bagwell, B. E., Wick, D., V., Batchko, R., Mansell, J. D., Martinez, T., Serati, S., Sharp, G., and Schwiegerling J., "Liquid crystal based active optics," Proc. SPIE 6289, 628908 (2006).

[6] Martinez, T., Wick ,D. V., Payne, D. M., Baker, J. T., and Restaino, S. R., "Non-mechanical zoom system," Proc. SPIE 5234, 375 (2004).

[7] Tam, E. C., "smart electro-optical zoom lens," Opt. Lett. 17, 369 (1992).

[8] Ye, M., Noguchi, M., Wang, B., and Sato,S., "Zoom lens system without moving elements realized using liquid crystal lenses," Elec. Lett. 45, 646 (2009).

[9] Valley, P., Dodge, M. R., Schwiegerling, J., Peyman, G., and Peyghambarian, N., "Nonmechanical bifocal zoom telescope," Opt. Lett. 35, 2582 (2010).

[10] Lin, H. C. and Lin, Y. H., "A fast response and large electrically tunable-focusing imaging system based on switching of two modes of a liquid crystal lens" Appl. Phys. Lett. 97, 063505 (2010).

[11] Lin, H. C. and Lin, Y. H., "An electrically tunable focusing pico-projector adopting a liquid crystal lens" J. Jpn. Appl. Phys. 49, 102502 (2010)

[12] Lin, Y. H., Chen, M. S., and Lin, H. C., "An electrically tunable optical zoom system using two composite liquid crystal lenses with a large zoom ratio," Opt. Express 19, 4714-4721 (2011).

[13] de Gennes, P.G. and Prost, J., [The physics of liquid crystal], Claredon Press, Oxford, (1993).

[14] Asatryan, K., Presnyakov, V., Tork, A., Zohrabyan, A., Bagramyan, A., and Galstian, T., "Optical lens with

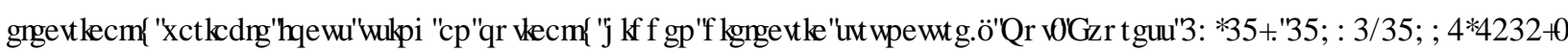

